\title{
Methylphenidate reduces functional connectivity of nucleus accumbens in brain reward circuit
}

\author{
J. G. Ramaekers • E. A. Evers • E. L. Theunissen • \\ K. P. C. Kuypers • A. Goulas • P. Stiers
}

Received: 14 November 2012 / Accepted: 27 March 2013

(C) Springer-Verlag Berlin Heidelberg 2013

\begin{abstract}
Release of dopamine in the nucleus accumbens (NAcc) is essential for acute drug reward. The present study was designed to trace the reinforcing effect of dopamine release by measuring the functional connectivity (FC) between the NAcc and brain regions involved in a limbic cortical-subcortical circuit during a dopaminergic challenge. Twenty healthy volunteers received single doses of methylphenidate $(40 \mathrm{mg}$ ) and placebo on separate test days according to a double-blind, cross-over study design. Resting state functional magnetic resonance imaging (fMRI) was measured between 1.5 and $2 \mathrm{~h}$ postdosing. FC between regions of interest (ROI) in the NAcc, the medial dorsal nucleus (MDN) of the thalamus and remote areas within the limbic circuit was explored. Methylphenidate significantly reduced FC between the NAcc and the basal ganglia (i.e., subthalamic nucleus and ventral pallidum (VP)), relative to placebo. Methylphenidate also decreased FC between the NAcc and the medial prefrontal cortex (mPFC) as well as the temporal cortex. Methylphenidate did not affect FC between MDN and the limbic circuit. It is concluded that methylphenidate directly affects the limbic reward circuit. Drug-induced changes in FC of the NAcc may serve as a useful marker of drug activity in in the brain reward circuit.
\end{abstract}

Keywords Drug abuse $\cdot$ Dopaminergic $\cdot$ Limbic circuit

Trial registration: http://www.trialregister.nl/trialreg/admin/ retview.asp?TC $=2033$.

J. G. Ramaekers $(\bowtie) \cdot$ E. A. Evers · E. L. Theunissen •

K. P. C. Kuypers $\cdot$ A. Goulas $\cdot$ P. Stiers

Department Neuropsychology \& Psychopharmacology, Faculty

of Psychology and Neuroscience, Maastricht University,

Maastricht, The Netherlands

e-mail: j.ramaekers@maastrichtuniversity.nl

\section{Introduction}

Dopamine transmission in the mesolimbic pathway has been associated with reward-seeking and impulsive behaviors, such as addiction (Pierce and Kumaresan 2006; Volkow et al. 2007). The mesolimbic dopamine pathway includes dopaminergic neurons in the ventral tegmental area (VTA) and its prime target in the limbic forebrain, the nucleus accumbens (NAcc). Drugs of abuse are believed to either directly or indirectly increase dopamine transmission in the NAcc (Nestler 2005). Activation of dopamine in the NAcc is essential for acute drug reward and can serve a useful brain marker of the addictive potential of drugs (Dagher and Robbins 2009; Kalivas and Volkow 2011; Volkow et al. 2011).

The mesolimbic dopamine pathway is tightly connected to a limbic "reward" circuitry in the brain. The latter has been described as a functionally segregated cortical-subcortical network that receives modulatory input to the NAcc from ascending dopaminergic projections from the VTA (Alexander et al. 1986; Bonelli and Cummings 2007; Cummings 1993; Perreault et al. 2011). The limbic circuit includes the medial prefrontal cortex (mPFC), amygdala, and hippocampus that send glutamatergic projections to the NAcc. The NAcc is believed to innervate the ventral pallidum (VP) either directly or indirectly through GABAergic and GABAergic/glutamatergic projections, respectively. The VP is not clearly divided in internal and external segments but reciprocal connections of the NAcc with the subthalamic nucleus (STN) have been identified that indicate the existence of direct and indirect pathways (Cummings 1993). Dopamine acting at D1 receptors in the NAcc results in stimulation of the direct pathway, while dopamine acting at $\mathrm{D} 2$ receptors results in inhibition of the indirect pathway. The VP sends GABAergic efferents to the medial dorsal nucleus (MDN) of the thalamus. Glutamatergic 
projections from the medial dorsal thalamus to the $\mathrm{mPFC}$ close the limbic circuit (Alexander et al. 1986; Bonelli and Cummings 2007; Cummings 1993; Perreault et al. 2011). Dopaminergic manipulation of neuronal communication is crucial to the normal functioning of the limbic brain circuit of reward. Neuronal communication within brain networks can be studied with functional connectivity (FC) in resting state functional magnetic resonance imaging (fMRI) by measuring signal synchronicity among remote brain areas within the network. Pharmacological fMRI research has shown that FC measures are sensitive to dopaminergic drugs that either increase or decrease dopamine transmission in the brain. In general, dopamine agonists and antagonists produced opposing effects. Drugs that increase dopamine transmission such as methylphenidate and L-dopa have been shown to increase FC in cognitive, attentional, and salience networks (Cole et al. 2012; Kelly et al. 2009), whereas dopamine antagonists such as haloperidol and sulpiride have been shown to decrease FC (Achard and Bullmore 2007; Cole et al. 2012). Alternatively, dopamine agonists and antagonists have also been reported to, respectively, decrease and increase FC in cortical-subcortical networks (Honey et al. 2003). Dopamine agonist and antagonist, thus, can decrease as well as increase FC in specific networks. The direction of change of FC is likely to depend on the locus of dopaminergic input within the network and subsequent interaction with additional neurotransmitter systems operating within the same neural network that may exhibit stimulatory or inhibitory actions.

The present study focused on the reinforcing effects of the psychostimulant methylphenidate within the limbic circuit. Methylphenidate promotes release of stored dopamine from presynaptic vesicles and blocks the return of dopamine into presynaptic nerve endings. Positron emission tomography (PET) studies have demonstrated that methylphenidate increases extracellular dopamine in the basal ganglia in a concentration-dependent manner (Volkow et al. 2002; Volkow et al. 2005a). We hypothesized that methylphenidate administration would increase dopamine concentrations in the NAcc and decrease FC of the NAcc within the limbic circuit by stimulation of GABAergic inhibitory projections in the direct pathway and by inhibition of the VP brake on GABAergic inhibitory projections to the STN in the indirect pathway. In other words, the key expectation was that increased inhibitory GABAergic output from the NAcc would also reflect as an inhibition or reduction in FC of the NAcc with other structures in the limbic circuit. In addition, we explored FC of brain areas within the limbic circuit, i.e., the MDN to assess the effects of methylphenidate on brain areas that are more remotely connected to the NAcc. FC was assessed in a placebo-controlled, double- blind, cross-over study in healthy volunteers that received single doses of placebo and methylphenidate $(40 \mathrm{mg})$.

\section{Methods}

Subjects

Twenty healthy, right-handed volunteers (nine males, 11 females) aged between 23 and 35 years of age were included. Volunteers were recruited through flyers distributed at Maastricht University. Initial screening comprised of a questionnaire on medical history. Subjects were examined by the medical supervisor who checked vital signs, conducted a resting 12-lead electrocardiogram (ECG), and took blood and urine samples. Standard blood chemistry, hematology, and drug screen tests were conducted on these samples. General inclusion criteria were: right handedness; free from psychotropic medication; good physical health as determined by medical examination and laboratory analysis; absence of any major medical, endocrine, and neurological condition; normal weight, body mass index (weight/length ${ }^{2}$ ) between 18 and $28 \mathrm{~kg} / \mathrm{m}^{2}$; and written informed consent. Exclusion criteria were: history of drug abuse as assessed by drug urine screens and questionnaires; pregnancy or lactation or failure to use reliable contraceptives; excessive drinking ( $>20$ standard alcoholic consumptions/beverages a week); hypertension (diastolic $>99$; systolic $>160$ ); magnetic resonance imaging (MRI) contraindications, such as claustrophobia and metal parts in the body; cardiovascular abnormalities; and history of psychiatric disorders.

The study was approved by the independent ethics committee of Maastricht University and conducted according to the code of ethics on human experimentation established by the Declaration of Helsinki (1964) and amended in Seoul (2008). All subjects were fully informed of study procedures, adverse reactions to drug treatments, legal rights and responsibilities, expected benefits of a general scientific nature, and their right for voluntary termination without penalty or censure.

Drugs, design, and procedures

The study was conducted according to a double-blind, placebo-controlled, two-way design. Volunteers received single oral doses of methylphenidate $(40 \mathrm{mg})$ and placebo. Treatments were counterbalanced across volunteers. Volunteers received treatments upon arrival at the laboratory. A blood sample was taken prior to scanning, i.e., at $70 \mathrm{~min}$ postdosing. Scanning sessions were conducted between 1.5 and $2 \mathrm{~h}$ postdosing and 
consisted of localizer scans, a T1 structural scan, and a resting state fMRI scan. Volunteers were not allowed to use alcohol on the day prior to an experimental session and were requested to arrive at experimental sessions well rested. Drug and alcohol screens were performed prior to experimental sessions after subject arrival at the site.

\section{Pharmacokinetics}

Plasma values of methylphenidate (micrograms per liter) and the metabolite ritalin acid (micrograms per liter) were determined with liquid chromatography-mass spectrometry. Limits of quantification for methylphenidate and ritalin acid were 1 and $10 \mu \mathrm{g} / \mathrm{l}$, respectively.

\section{Image acquisition}

MRI images were acquired with a Siemens $3 \mathrm{~T}$ head-only scanner (MAGNETOM Allegra, Siemens Medical Systems, Erlangen, Germany). During resting state, we acquired 200 EPI whole-brain functional volumes $(\mathrm{TR}=2.0 \mathrm{~s}$; $\mathrm{TE}=$ $30 \mathrm{~ms}$; field of view $(\mathrm{FOV})=224 \times 224 \mathrm{~mm}$; flip angle $=90^{\circ}$; oblique acquisition orientation; interleaved slice acquisition; 32 slices; slice thickness $=3.5 \mathrm{~mm}$; image matrix $=64 \times 64 \times$ 32; voxel size $=3.5 \times 3.5 \times 3.5 \mathrm{~mm}$ ). During resting state scans, volunteers looked at a blank screen and were asked to relax with the eyes open. The T1-weighted anatomical scan was acquired with the following parameters: $\mathrm{TR}=$ $2,250 \mathrm{~ms}$; TE $=2.6 \mathrm{~ms}$; flip angle $=9^{\circ}$; image matrix $=196 \times$ $256 \times 256,192$ sagittal slices, voxel size $=1 \times 1 \times 1 \mathrm{~mm}$ ).

\section{Image preprocessing}

Data processing and analysis were conducted using SPM5. The first two volumes were removed from each fMRI data set to allow for magnetic equilibration. Preprocessing consisted of 3D motion correction and slice time correction. Individual anatomical data sets were normalized to standard 3-D MNI space. Individual functional images were realigned, coregistered and normalized to the anatomical data, and resampled to a voxel size of $2 \times 2 \times 2 \mathrm{~mm}^{3}$. Spatial smoothing was conducted with a FWHM $6 \mathrm{~mm}$ Gaussian kernel.

\section{Functional connectivity}

FC data were produced with the MATLAB toolbox DPARSF (Chao-Gan and Yu-Feng 2010) according to the following processing steps. First, linear trends of time courses were removed followed by low band-pass filtering $(0.01-0.08 \mathrm{~Hz})$ of the preprocessed data to remove "noise" attributable to physiological parameters. In addition, nuisance covariates, including six motion parameters, and the average signal of white matter voxels and of cerebral spinal fluid (CSF) voxels were removed though linear regression. White matter and CSF voxels were selected based on the tissue density maps derived from segmenting the anatomical image. For all voxels with a density of higher than 90 and $70 \%$, respectively, the time series data were voxel-wise averaged. No global brain signals were removed (Murphy et al. 2009). Four spheres (radius $4 \mathrm{~mm}$ ) were created that were located (in MNI space) in the NAcc of the left $(-9,9,-9)$ and right $(9,9,-9)$ hemispheres, and the MDN of the thalamus in the left $(-9,-19,-6)$ and right $(9,-19,-6)$ hemispheres. Seeds locations were in agreement with structural and functional subdivisions of these brain regions that were validated in previous work for the probability of belonging to a given anatomical region (Di Martino et al. 2008; Kelly et al. 2009; McMahon et al. 2006). Average time courses were obtained for each sphere region of interest (ROI) and correlational analysis was performed voxelwise to generate FC maps for each sphere ROI. Finally, the correlation coefficient map was converted into z-maps by Fisher's r-to-z transform to improve normality.

\section{Statistical analysis}

Statistical analyses were conducted using SPM5. One sample $t$ tests were performed on z-maps for methylphenidate and placebo separately to show significant FC patterns associated with ROI seeds in each treatment condition. The within-condition statistical threshold was set at $p<$ 0.05 FWE corrected (i.e., corresponding to $T>7.50$ ). FC maps for both treatments were subsequently modeled in a repeated measures factorial design to contrast significant differences in FC between methylphenidate and placebo. The threshold limit for methylphenidate-placebo contrasts was set at $p<0.05$ (FDR corrected, cluster size $>10$ ).

\section{Results}

Figures 1 and 2 show significant FC data in each treatment condition for each seed ROI as indicated by one-sample $t$ tests. In addition, the figures indicate significant differences in FC between treatments as indicated by placebo-methylphenidate contrasts.

FC of the NAcc showed high synchronicity with large areas in basal ganglia, thalamus, amygdala, hippocampus, brainstem, and temporal and frontal cortices during placebo. Treatment with methylphenidate significantly reduced $\mathrm{FC}$ in a number of these areas. A summary of the major clusters showing decrements in FC with the NAcc in the left and right hemispheres is given in Table 1. The clusters include areas that are part of the limbic circuit such as VP and STN as well the mPFC. 
Nucleus accumbens: left hemisphere ( $\mathrm{MNI}$ seed $-9,9,-9$ )
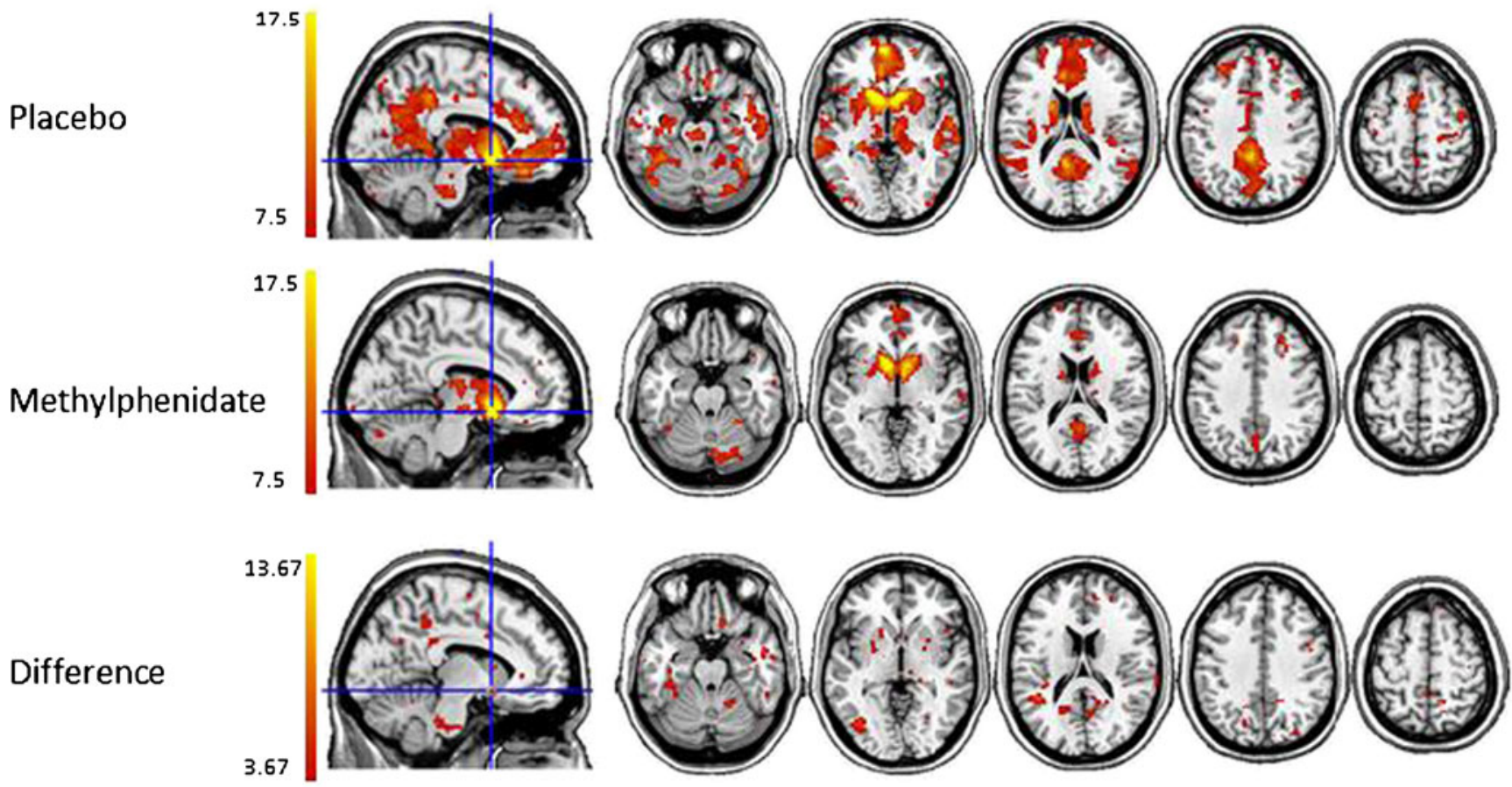

Nucleus accumbens: right hemisphere (MNI seed 9,9,-9)

Placebo
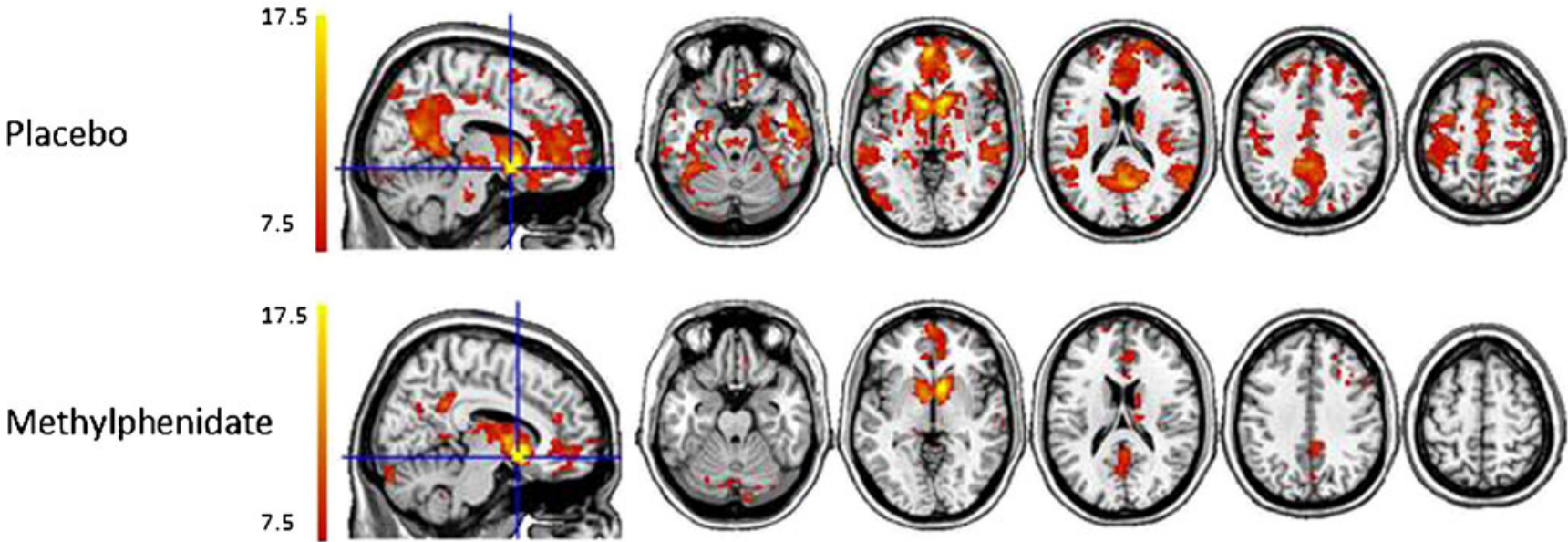

Difference

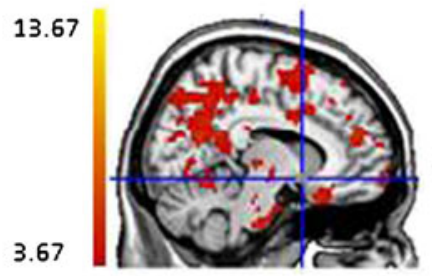

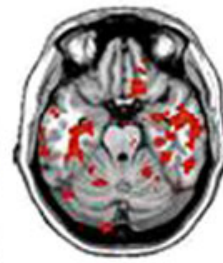

$z=-22$

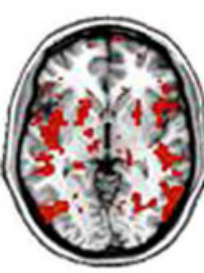

$z=-2$

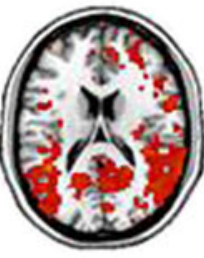

$z=12$
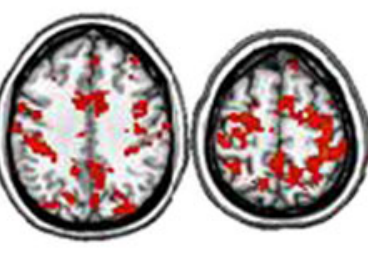

$z=38$

$z=58$

Fig. 1 NAcc-related functional connectivity in the left and right hemispheres. Shown are thresholded Z-score maps of functional connectivity for each condition and significant methylphenidate-related decrements in FC. The cross-hair indicates the seed ROI position (axial orientation: left=left) 
Medial Dorsal Nucleus: left hemisphere (MNI seed $-9,-18,-6$ )

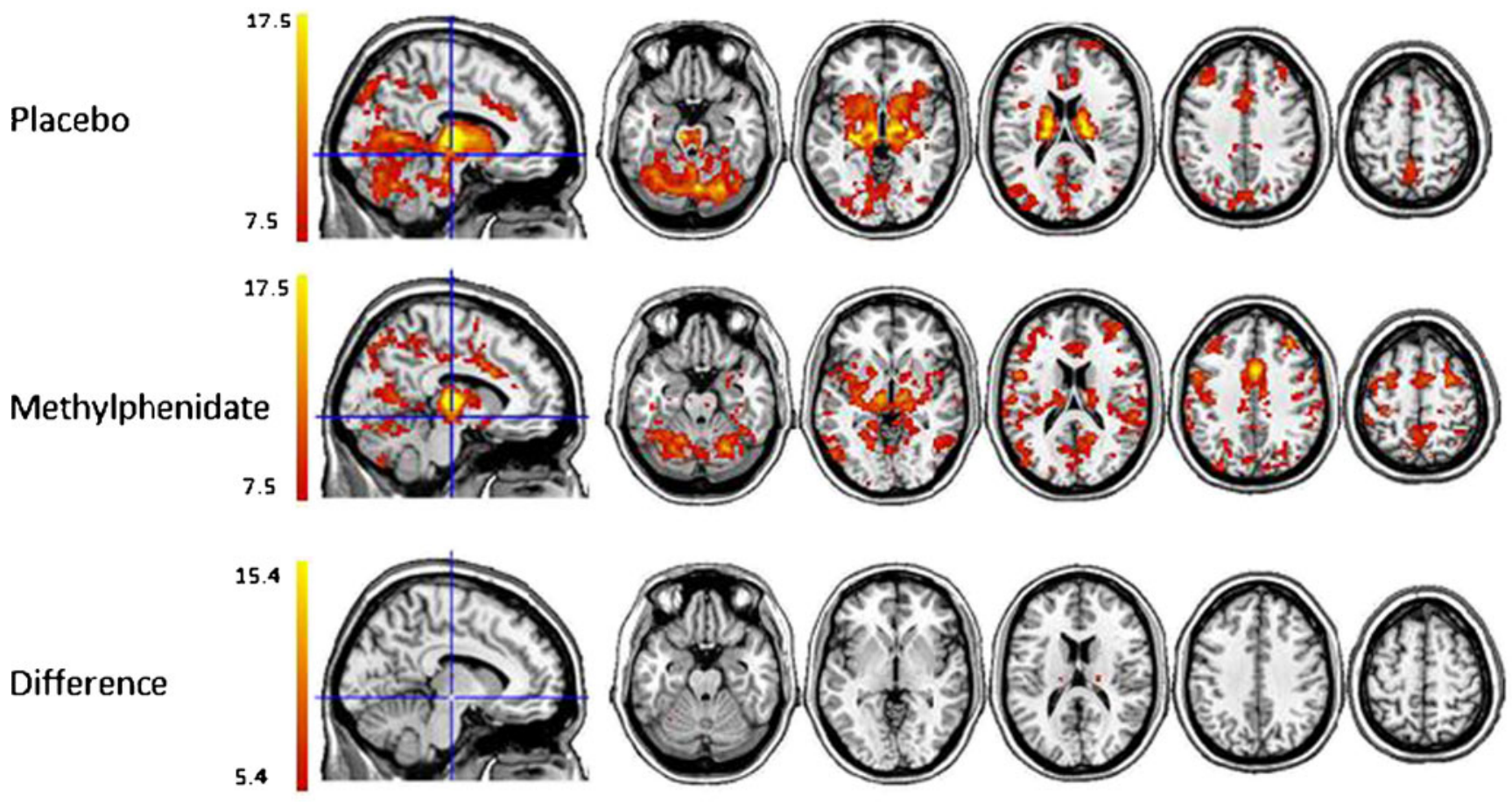

Medial Dorsal Nucleus: right hemisphere (MNI seed 9, -18,-6)
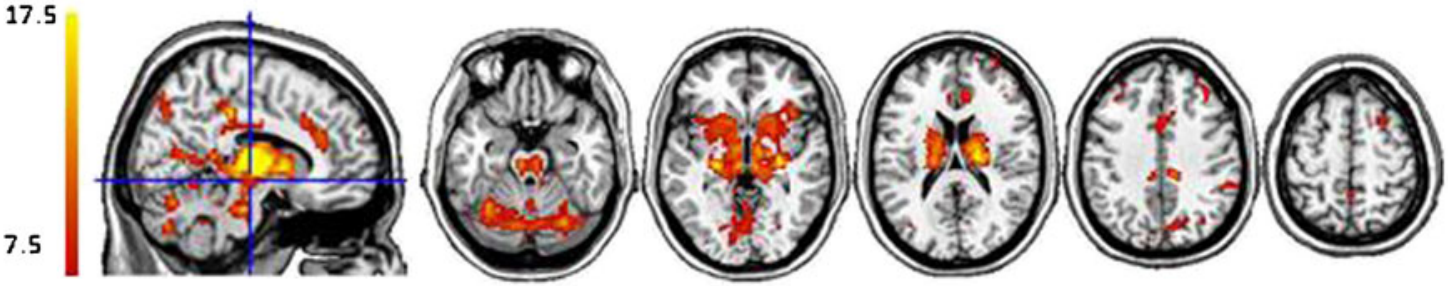

Placebo
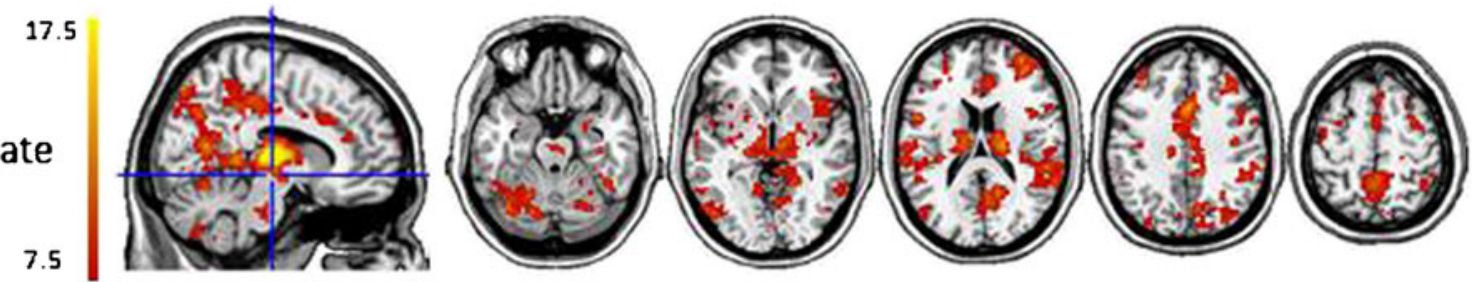

Methylphenidate

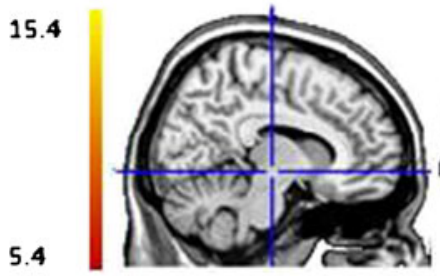

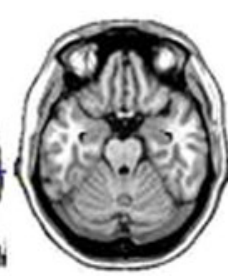

$z=-22$

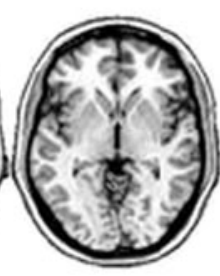

$z=-2$

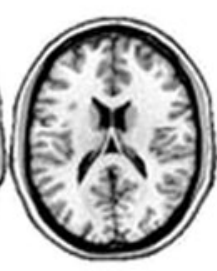

$z=12$

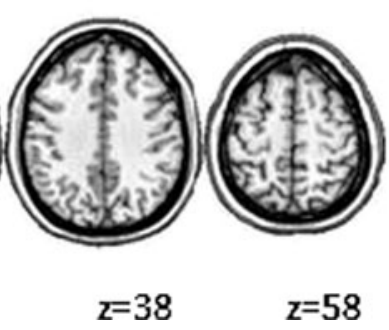

Fig. 2 MDN of the thalamus-related functional connectivity in the left and right hemispheres. Shown are thresholded $Z$-score maps of functional connectivity for each condition and significant methylphenidate-related decrements in FC. The cross-hair indicates the seed ROI position (axial orientation: left=left) 
Table 1 Significant decrements in functional connectivity of nucleus accumbens and medial dorsal nucleus for methylphenidate, relative to placebo

\begin{tabular}{|c|c|c|c|c|}
\hline Brain clusters & BA & $\begin{array}{l}\text { Number } \\
\text { of voxels }\end{array}$ & $\begin{array}{l}\text { Peak MNI } \\
\text { coordinates }\end{array}$ & $\begin{array}{l}P \text { value } T_{19}, \\
\text { FDR corrected }\end{array}$ \\
\hline \multicolumn{5}{|l|}{ Seed: right nucleus accumbens } \\
\hline Right medial frontal gyrus, right superior frontal gyrus, anterior cingulate & 10,32 & 115 & $14,68,-12$ & 0.009 \\
\hline Right middle temporal gyrus & $19,20,21,39$ & 30,787 & $54,-68,24$ & 0.003 \\
\hline Right brainstem, subthalamic nucleus, medial globus pallidus, ventral pallidum & & 177 & $6,-12,-8$ & 0.021 \\
\hline Left middle frontal gyrus & 8,9 & 270 & $-24,28,28$ & 0.015 \\
\hline Left superior temporal gyrus & & 165 & $-42,-48,18$ & 0.003 \\
\hline Left brain stem, left cerebellum, subthalamic nucleus & & 293 & $-12,-26,-40$ & 0.013 \\
\hline \multicolumn{5}{|l|}{ Seed: left nucleus accumbens } \\
\hline Right precuneus & 7,31 & 265 & $8,-58,48$ & 0.026 \\
\hline Right posterior cingulate & 23,29 & 233 & $6,-62,20$ & 0.027 \\
\hline Right cerebellum & & 133 & $18,-54,-20$ & 0.021 \\
\hline Left superior temporal gyrus, left fusiform gyrus & 20 & 165 & $-42,-48,18$ & 0.008 \\
\hline Left precuneus, left posterior cingulate, left cuneus & $19,30,31$ & 276 & $-22,-70,32$ & 0.021 \\
\hline Left inferior occipital gyrus, left fusiform gyrus & 19 & 173 & $-40,-72,-2$ & 0.021 \\
\hline Left brain stem, left cerebellum & & 325 & $-12,-30,-38$ & 0.023 \\
\hline \multicolumn{5}{|l|}{ Seed: right medial dorsal nucleus } \\
\hline Right thalamus & & & $18,-20,16$ & 0.035 \\
\hline Left thalamus & & & $-8,-16,10$ & 0.036 \\
\hline
\end{tabular}

FC of the MDN showed strong correlations between seed ROIs and brain areas in the basal ganglia, parietal, and temporal and frontal cortices. Treatment with methylphenidate somewhat decreased FC within the thalamic structures in both hemispheres, but FC with all other brain areas in the network was unaffected. Plasma data revealed mean (SD) methylphenidate and ritalinic acid concentrations of 7.1 (6.8) and $265.2(262.1) \mu \mathrm{g} / \mathrm{l}$, respectively.

\section{Discussion}

The present study was designed to assess methylphenidateinduced modulation of FC within the limbic circuit. We hypothesized that methylphenidate administration would increase dopamine concentrations in the NAcc and decrease FC of the NAcc within the limbic circuit by stimulation of GABAergic inhibitory projections in the limbic circuit. FC data obtained during treatment with placebo demonstrated that NAcc activity was strongly correlated to a large number of brain areas including those that are involved in the limbic circuit. The present data are supportive of previous FC studies in healthy volunteers that have also shown that spontaneous activity in NAcc predicts activity in regions implicated in the limbic circuit (Cauda et al. 2011). Treatment with methylphenidate significantly affected FC of the NAcc within the direct and indirect pathways of the basal ganglia as indicated by a reduction in synchronicity between the NAcc and the VP and between the NAcc and the STN. In addition, methylphenidate also decreased FC between the NAcc and the medial PFC (e.g., Brodmann areas 9, 10, and 32) and temporal cortex (e.g., Brodmann area 20). These data strongly confirm that methylphenidate directly affects the limbic reward circuit and decreases FC of brain areas within this network.

Decrements in FC of the NAcc may be largely related to the moderating effects of methylphenidate on GABAergic projections to output nuclei within the basal ganglia. Methylphenidate elevates dopamine levels in the NAcc, which in turn, increases inhibitory GABAergic neurotransmission to the VP through D2 receptor stimulation in the direct pathway and decreases stimulatory glutamatergic neurotransmission to VP by blocking GABAergic output through D1 receptor stimulation in the indirect pathway. The striatal complex has previously been described as an bottom-up "impulse" generator within the limbic circuit that signals pleasure and reward to the medial prefrontal brain (Bechara 2005). Such pleasure signals appear tightly linked to GABA pathways. Microinjections of GABA agonists in the NAcc have been shown to amplify "liking" of drugs, whereas glutamatergic signals failed to alter pleasure reactions in rats (Faure et al. 2010). Likewise, chronic drug abuse in humans has been associated with a disruption of GABA pathways in the striatum and increased sensitivity to GABA agonists (Volkow et al. 1998). Methylphenidate has also been reported to increase drug "liking" after intravenous 
dosing and, to lesser degree, after therapeutic oral doses (Kollins et al. 2009; Volkow et al. 1999), depending on rate of drug clearance in the striatum (Parasrampuria et al. 2007; Spencer et al. 2006). Together, these findings suggest that drug-induced reductions in FC between NAcc and VP or STN following altered processing of dopaminergic signals in GABAergic pathways may serve as striatal markers of reinforcement during drug treatment. Additional research, however, is needed to quantify and validate the relation between drug "liking" and FC measures in striatum.

The mPFC has been associated with "reflective" or "topdown" control in the limbic circuit that receives glutamatergic input from the MDN of the thalamus and produces glutamatergic output to the NAcc (Bechara 2005; Faure et al. 2010; Kalivas et al. 2005; Kalivas and Volkow 2005). Topdown cortical glutamate signals regulate motivational components of drug use but may be relatively unable to affect the hedonic component of drug use (Kalivas and Volkow 2005). Methylphenidate reduced FC between the NAcc and the $\mathrm{mPFC}$ as well as temporal brain areas. The reduction in FC seems to imply a loss of connectivity between the reflective and the impulsive parts of the limbic circuit. Reductions in FC between the NAcc and the MPFC have also been reported in heroin- (Hong et al. 2009) and cocaine-dependent (Gu et al. 2010; Tomasi et al. 2010) individuals. Reductions in FC in these studies have been interpreted as additional support for the general notion of PFC dysfunction in drug addicts underlying loss of self-control (Goldstein and Volkow 2011a). The reduction in FC between NAcc and $\mathrm{mPFC}$ after a single dose of methylphenidate appears to contrasts with recent imaging findings that this drug normalizes activity in the $\mathrm{mPFC}$ and increases impulse control in cocaine abusers (Goldstein and Volkow 2011b). Yet, previous studies have also shown that methylphenidate can produce contrasting responses in healthy controls and drug addicts. Intravenous administration of methylphenidate increased brain metabolism in cocaine addicts but decreased metabolism in healthy controls (Volkow et al. 2005b). These data suggest that the brain's response to methylphenidate varies with (ab)normal fluctuations of glutamatergic and GABAergic neurotransmission in the limbic circuit. The latter implies a strong potential for FC measures to assess neuroadaptions in neurotransmission within the limbic circuit by comparing acute drug effects on FC in occasional drug users and in chronic drug users.

The finding that methylphenidate decreases FC of the NAcc within the limbic circuit seems generally in line with data from other placebo-controlled studies in healthy volunteers that also reported on FC in cortical-striatal networks during drug treatments that increase dopamine levels in the striatum. L-Dopa $(100 \mathrm{mg})$ decreased FC between the ventral striatum (NAcc) and temporal and occipital brain areas in one study (Kelly et al. 2009). The same drug, however, failed to affect $\mathrm{FC}$ between the ventral striatum and $\mathrm{mPFC}$ when compared to placebo in another study (Cole et al. 2012). Methylphenidate $(20 \mathrm{mg})$ reduced FC between the dorsal striatum and the thalamus but not between dorsal striatum and (m)PFC (Honey et al. 2003). Some of the discrepancies in these studies may be related to differences in drug doses, dopamine availability in the NAcc (Carboni et al. 2003; Chong et al. 2012; Podet et al. 2010), or drug plasma concentration that are known to vary considerably between individuals even when receiving identical doses. In the present study, mean plasma data concentrations of methylphenidate and ritalinic acid concentrations were well within the expected therapeutic range (Lee et al. 2003), but they may have been higher as compared to previous FC studies using a lower dose.

FC of MDN of the thalamus revealed significant correlations between MDN and striatum as well as frontal, temporal, and occipital brain areas during placebo treatment. Methylphenidate, however, did not alter the FC of MDN. The only notable change was a small reduction in $\mathrm{FC}$ within the thalamic nuclei. This general lack of change in FC of the MDN may be related to the fact that this structure is more distal and not directly connected to the target site of methylphenidate, i.e., the NAcc. FC of the MDN within the limbic circuit, thus, may remain relatively stable during treatment with dopaminergic drugs such as methylphenidate, whereas FC of brain areas that are closely connected to the target site of dopaminergic drugs such as the VP, STN, and mPFC may strongly decrease.

In sum, the present study demonstrated that methylphenidate directly affects the limbic reward circuit and decreases FC between the NAcc, basal ganglia, and mPFC. Drug-induced changes in FC of the NAcc may serve as a useful marker of drug activity in the brain reward circuit.

\section{References}

Achard S, Bullmore E (2007) Efficiency and cost of economical brain functional networks. PLoS Comput Biol 3:e17

Alexander GE, DeLong MR, Strick PL (1986) Parallel organization of functionally segregated circuits linking basal ganglia and cortex. Annu Rev Neurosci 9:357-381

Bechara A (2005) Decision making, impulse control and loss of willpower to resist drugs: a neurocognitive perspective. Nat Neurosci 8:1458-1463

Bonelli RM, Cummings JL (2007) Frontal-subcortical circuitry and behavior. Dialogues Clin Neurosci 9:141-151

Carboni E, Silvagni A, Valentini V, Di Chiara G (2003) Effect of amphetamine, cocaine and depolarization by high potassium on extracellular dopamine in the nucleus accumbens shell of SHR rats. An in vivo microdyalisis study. Neurosci Biobehav Rev 27:653-659

Cauda F, Cavanna AE, D'Agata F, Sacco K, Duca S, Geminiani GC (2011) Functional connectivity and coactivation of the nucleus accumbens: a combined functional connectivity and structurebased meta-analysis. J Cogn Neurosci 23:2864-2877 
Chao-Gan Y, Yu-Feng Z (2010) DPARSF: a MATLAB toolbox for "pipeline" data analysis of resting-state fMRI. Front Syst Neurosci 4:13

Chong SL, Claussen CM, Dafny N (2012) Nucleus accumbens neuronal activity in freely behaving rats is modulated following acute and chronic methylphenidate administration. Brain Res Bull 87:445-456

Cole DM, Oei NY, Soeter RP, Both S, van Gerven JM, Rombouts SA, Beckmann CF (2012) Dopamine-dependent architecture of corticosubcortical network connectivity. Cerebr Cortex. doi:10.1093/ cercor/bhs 136

Cummings JL (1993) Frontal-subcortical circuits and human behavior. Arch Neurol 50:873-880

Dagher A, Robbins TW (2009) Personality, addiction, dopamine: insights from Parkinson's disease. Neuron 61:502-510

Di Martino A, Scheres A, Margulies DS, Kelly AM, Uddin LQ, Shehzad Z, Biswal B, Walters JR, Castellanos FX, Milham MP (2008) Functional connectivity of human striatum: a resting state FMRI study. Cerebr Cortex 18:2735-2747

Faure A, Richard JM, Berridge KC (2010) Desire and dread from the nucleus accumbens: cortical glutamate and subcortical GABA differentially generate motivation and hedonic impact in the rat. PLoS One 5:e11223

Goldstein RZ, Volkow ND (2011a) Dysfunction of the prefrontal cortex in addiction: neuroimaging findings and clinical implications. Nat Rev Neurosci 12:652-669

Goldstein RZ, Volkow ND (2011b) Oral methylphenidate normalizes cingulate activity and decreases impulsivity in cocaine addiction during an emotionally salient cognitive task. Neuropsychopharmacol Off Publ Am Coll Neuropsychopharmacol 36:366-367

Gu H, Salmeron BJ, Ross TJ, Geng X, Zhan W, Stein EA, Yang Y (2010) Mesocorticolimbic circuits are impaired in chronic cocaine users as demonstrated by resting-state functional connectivity. NeuroImage 53:593-601

Honey GD, Suckling J, Zelaya F, Long C, Routledge C, Jackson S, Ng V, Fletcher PC, Williams SC, Brown J, Bullmore ET (2003) Dopaminergic drug effects on physiological connectivity in a human cortico-striato-thalamic system. Brain J Neurol 126:1767-1781

Hong LE, Gu H, Yang Y, Ross TJ, Salmeron BJ, Buchholz B, Thaker GK, Stein EA (2009) Association of nicotine addiction and nicotine's actions with separate cingulate cortex functional circuits. Arch Gen Psychiatry 66:431-441

Kalivas PW, Volkow N, Seamans J (2005) Unmanageable motivation in addiction: a pathology in prefrontal-accumbens glutamate transmission. Neuron 45:647-650

Kalivas PW, Volkow ND (2005) The neural basis of addiction: a pathology of motivation and choice. Am J Psychiatry 162:1403-1413

Kalivas PW, Volkow ND (2011) New medications for drug addiction hiding in glutamatergic neuroplasticity. Mol Psychiatry 16:974-986

Kelly C, de Zubicaray G, Di Martino A, Copland DA, Reiss PT, Klein DF, Castellanos FX, Milham MP, McMahon K (2009) L-Dopa modulates functional connectivity in striatal cognitive and motor networks: a double-blind placebo-controlled study. J Neurosci Off J Soc Neurosci 29:7364-7378

Kollins SH, English J, Robinson R, Hallyburton M, Chrisman AK (2009) Reinforcing and subjective effects of methylphenidate in adults with and without attention deficit hyperactivity disorder (ADHD). Psychopharmacology 204:73-83

Lee L, Kepple J, Wang Y, Freestone S, Bakhtiar R, Hossain M (2003) Bioavailability of modified-release methylphenidate: influence of high-fat breakfast when administered intact and when capsule content sprinkled on applesauce. Biopharm Drug Dispos 24:233-243
McMahon K, Copland DA, De Zubicaray G, MB (2006) Effects of levodopa administration on cerebral functional connectivity 12th Annual Meeting of the Organization for Human Brain Mapping. . Florence, Italy

Murphy K, Birn RM, Handwerker DA, Jones TB, Bandettini PA (2009) The impact of global signal regression on resting state correlations: are anti-correlated networks introduced? NeuroImage 44:893-905

Nestler EJ (2005) Is there a common molecular pathway for addiction? Nat Neurosci 8:1445-1449

Parasrampuria DA, Schoedel KA, Schuller R, Silber SA, Ciccone PE, $\mathrm{Gu}$ J, Sellers EM (2007) Do formulation differences alter abuse liability of methylphenidate? A placebo-controlled, randomized, double-blind, crossover study in recreational drug users. J Clin Psychopharmacol 27:459-467

Perreault ML, Hasbi A, O'Dowd BF, George SR (2011) The dopamine $\mathrm{d} 1-\mathrm{d} 2$ receptor heteromer in striatal medium spiny neurons: evidence for a third distinct neuronal pathway in Basal Ganglia. Front Neuroanat 5:31

Pierce RC, Kumaresan V (2006) The mesolimbic dopamine system: the final common pathway for the reinforcing effect of drugs of abuse? Neurosci Biobehav Rev 30:215-238

Podet A, Lee MJ, Swann AC, Dafny N (2010) Nucleus accumbens lesions modulate the effects of methylphenidate. Brain Res Bull 82:293-301

Spencer TJ, Biederman J, Ciccone PE, Madras BK, Dougherty DD, Bonab AA, Livni E, Parasrampuria DA, Fischman AJ (2006) PET study examining pharmacokinetics, detection and likeability, and dopamine transporter receptor occupancy of short- and long-acting oral methylphenidate. Am J Psychiatry 163:387-395

Tomasi D, Volkow ND, Wang R, Carrillo JH, Maloney T, Alia-Klein N, Woicik PA, Telang F, Goldstein RZ (2010) Disrupted functional connectivity with dopaminergic midbrain in cocaine abusers. PLoS One 5:e10815

Volkow ND, Fowler JS, Wang G, Ding Y, Gatley SJ (2002) Mechanism of action of methylphenidate: insights from PET imaging studies. J Atten Disord 6(Suppl 1):S31-S43

Volkow ND, Fowler JS, Wang GJ, Swanson JM, Telang F (2007) Dopamine in drug abuse and addiction: results of imaging studies and treatment implications. Arch Neurol 64:1575-1579

Volkow ND, Wang GJ, Fowler JS, Ding YS (2005a) Imaging the effects of methylphenidate on brain dopamine: new model on its therapeutic actions for attention-deficit/hyperactivity disorder. Biol Psychiatry 57:1410-1415

Volkow ND, Wang GJ, Fowler JS, Hitzemann R, Gatley SJ, Dewey SS, Pappas N (1998) Enhanced sensitivity to benzodiazepines in active cocaine-abusing subjects: a PET study. Am J Psychiatry 155:200-206

Volkow ND, Wang GJ, Fowler JS, Logan J, Gatley SJ, Gifford A, Hitzemann R, Ding YS, Pappas N (1999) Prediction of reinforcing responses to psychostimulants in humans by brain dopamine D2 receptor levels. Am J Psychiatry 156:1440-1443

Volkow ND, Wang GJ, Fowler JS, Tomasi D, Telang F (2011) Addiction: beyond dopamine reward circuitry. Proc Natl Acad Sci USA 108:15037-15042

Volkow ND, Wang GJ, Ma Y, Fowler JS, Wong C, Ding YS, Hitzemann R, Swanson JM, Kalivas P (2005b) Activation of orbital and medial prefrontal cortex by methylphenidate in cocaine-addicted subjects but not in controls: relevance to addiction. J Neurosci Off J Soc Neurosci 25:3932-3939 